\title{
La experiencia de Academia. Revista sobre la Enseñanza del Derecho en la constitución de un espacio de reflexión sobre la práctica docente en la UBA.
}

\section{The experience of Academia. Review on legal education in the constitution of an area of reflection on teaching practice in the UBA.}

Malvina Zacari $^{1}$

Resumen: El texto que sigue reflexiona sobre el desarrollo de la Revista Academia de la Facultad de Derecho de la Universidad de Buenos Aires, dando cuenta de la experiencia acumulada por la Revista Academia, desde los desafíos de su gestación hasta su consolidación como un espacio académico de discusión sobre modelos de formación jurídica.

El artículo pone en relevancia el impacto que tiene para los docentes contar con un lugar donde puedan reflexionar sobre sus propias prácticas. Puesto que, en tanto no se expliciten las estrategias, cambios e innovaciones implementadas en su desempeño, continuará vigente en el imaginario la hipótesis de enseñanza de un docente "dictando" contenidos y de estudiantes pasivos que toman apuntes para luego estudiarlos ( $\mathrm{y}$ repetirlos) en forma "memorística".

Palabras Clave: Revista Académica, enseñanza del derecho, pedagogía universitaria.

\begin{abstract}
:
This article reflects upon the development of Revista Academia, an academic journal published by the School of Law at the University of Buenos Aires. The article describes the accumulated experience through the history of the journal since its challenging beginning until its consolidation as a recognized academic space to discuss about different models for teaching law.

The article shows that is important to have a place in which teachers can think about their practice, if the strategies, changes and innovations used by teachers are not explicit and shared, the idea about teachers "dictating" contents to passive students that only take notes and repeat what is being said, will prevail in the collective imagination.
\end{abstract}

Key words: Academic journal, teaching law, university pedagogy.

\section{Introducción}

Este trabajo toma como objeto de investigación aquellas revistas que, desde su título u objetivos, se "enuncian" como revistas ${ }^{2}$ sobre enseñanza del Derecho. Exploramos la pregunta general acerca de cómo o en qué medida estas revistas pueden provocar, ayudar, sostener, dificultar, un proceso de discusión, actualización y-o implementación del currículum.

Partimos de dos presupuestos. Muchos creen que una reforma a un plan de estudios termina cuando se aprueban las normas que lo establecen. Sin embargo, el desafío

\footnotetext{
${ }^{1}$ Académica Facultad de Derecho, Universidad de Buenos Aires malvina1977@yahoo.com

${ }^{2}$ Toma como objeto de estudio a las revistas jurídicas en el contexto latinoamericano, Parise, A., "Las revistas jurídicas en el ámbito universitario: foros de expresión y laboratorios de escritura", en: Academia. Revista sobre enseñanza del Derecho, Buenos Aires, Nro. 15, 2010.
} 
continúa el día después. Un cambio de plan de estudios suele generar temores, por ejemplo, de los docentes que creen que "sus" asignaturas pierden importancia en el nuevo diseño curricular y que, por ende, ellos pierden "poder" en la formación de abogados. Así, parte de la planificación estratégica de implementación de un plan de estudios implica algún trabajo con los docentes que reduzca la resistencia y evite que se siga haciendo más o menos lo mismo llamándolo de otra forma. Segundo, las reformas a los planes de estudios que se plantean como objetivos, entre otros, generar pensamiento crítico en los estudiantes, requieren "conmover" las prácticas de enseñanza.

Así planteado, se pretende indagar en qué medida una revista sobre enseñanza del Derecho ayuda a generar ese espacio para los docentes. Nos interesa identificar sobre qué escriben, por ejemplo, cuáles temas aparecen en forma mayoritaria y continua, cuáles son marginales, cuáles se mantienen ausentes.

Nos detendremos en el análisis de un caso: el de la Revista Academia sobre Enseñanza del Derecho publicada por la Facultad de Derecho de la Universidad de Buenos Aires.

\section{Etapa de creación de Academia. Revista sobre enseñanza del Derecho}

Para desarrollar este punto nos remontaremos a los momentos previos a la creación de la revista Academia. Revista sobre Enseñanza del Derecho publicada por el Departamento de Publicaciones de la Facultad de Derecho de la Universidad de Buenos Aires. Realizaremos un mínimo recorrido sobre la etapa de conformación de la revista. En relación a ello nos resulta oportuno y valioso citar algunas palabras de nuestra actual decana de la Facultad, la Dra. Mónica Pinto, expresadas en una nota realizada el año 2010, al respecto de la memoria y la democracia "Prácticamente todas las civilizaciones han guardado registro de su evolución, de sus experiencias positivas y negativas. Si las primeras se registran con la finalidad pedagógica de resaltar los valores compartidos, las segundas apuntan a las lecciones aprendidas y, en general, se proponen el "Nunca Más". La evocación regular de estos eventos y sus enseñanzas constituyen una parte de la memoria de los pueblos". ${ }^{3}$ En este sentido entendemos que la memoria en el ámbito de la Enseñanza del Derecho, salvando las distancias del contexto en cual fueron esbozadas estas palabras, es importante evocarla a través de eventos que permiten recordar cómo se fue gestando y delineando en este caso el camino de surgimiento de la revista Academia. Revista sobre enseñanza del Derecho.

En esta línea nos resulta positivo rememorar entonces como primer acontecimiento previo a la creación de la revista, la presentación del libro "La Teoría de las Ficciones" de Enrique Marí en la Facultad de Ciencias Sociales de Universidad de Buenos Aires. ${ }^{4}$ Ese evento es el acontecimiento en dónde por primera vez Academia tuvo voz. En aquella oportunidad, la Directora del Departamento de Publicaciones de la Facultad de Derecho de la Universidad de Buenos Aires, Mary Beloff, nos anunciaba por primera

\footnotetext{
3 http://www.derecho.uba.ar/institucional/lafacultadenlosmedios/20100325_nota_memoria-democraciapagina12.php

4 La presentación del libro "La teoría de las ficciones" de Enrique Marí se realizó el 14 de noviembre de 2002 a las 19 hs en la Facultad de Ciencias Sociales, ver "Derecho al día" año I, nº 18, del 7 de noviembre del 2002, Departamento de Publicaciones, Facultad de Derecho, UBA.
} 
vez que crearíamos un proyecto sobre Enseñanza del Derecho para la Facultad de Derecho. También, alrededor de este recuerdo gira todo el esfuerzo y entusiasmo académico que provocó aquel proyecto. Academia fue para nosotras el primer proyecto académico sobre Enseñanza del Derecho dentro del Departamento de Publicaciones que nos involucraba como agentes de su gestación. En aquel entonces la revista se asomaba tan tímidamente que daba la sensación que en vez de acercarse el momento de su publicación se alejaba cada vez más.

El proyecto de resolución que debía darle forma concreta a la revista de Enseñanza del Derecho recorrió todas las instancias administrativas dentro de las secretarias pertinentes de la Facultad. Luego de ese arduo recorrido finalmente se publicó la augurada resolución de creación de la revista Academia.

Fue el 20 de diciembre de 2002 por medio de la Resolución (D) n ${ }^{\circ} 1892 / 2002$ que se creó formalmente la revista Academia. Revista sobre enseñanza del Derecho. Esta resolución le daba nacimiento e institucionalidad a la revista, su primer considerando expresaba sintéticamente que la revista "...tendrá como objeto, la difusión de ensayos, monografías, investigaciones, estudios, comentarios de legislación, bibliografía y jurisprudencia, vinculados con la enseñanza del derecho tanto en el ámbito de la Facultad, cuanto nacional, latinoamericano cómo internacional, que puedan interesar para un mejor desarrollo de las actividades docentes y de investigación". Así, con mucho esfuerzo, trabajo, entusiasmo y compromiso del Departamento de Publicaciones de la Facultad de Derecho de la Universidad de Buenos Aires nació Academia. Revista sobre enseñanza del Derecho.

Cómo comenzó a materializarse y cómo se robusteció la vida de la revista forma parte de otra etapa de producción del trabajo de Academia, esta fue y sigue siendo una etapa rica, llena de aprendizajes no sólo a nivel académico sino también en cuanto aspectos que van del más simple compromiso con el compañero de trabajo hasta los más complejos problemas que se encuentran alrededor del proceso de edición. Hay un largo recorrido entre los primeros paper y la publicación final pues en ellos no sólo están involucrados los autores, sino también los redactores, editores, traductores, diseñadores y autoridades del Departamento de Publicaciones, y todos ellos comprenden y contienen el vertiginoso proceso de producción de una revista tan notable como Academia. Si bien, describir e introducirnos en este proceso de producción de la revista es casi una tentación dado que está colmada de aprendizaje y enriquecimiento intelectual, hacerlo haría sumamente extenso este trabajo. Simplemente mencionaremos que todo comenzó a materializarse con la contribución de los primeros paper y trabajos que realizaron los profesores de nuestra Facultad de Derecho y de a poco se fueron conformando sus primeros volúmenes.

\section{Alcance y objetivos de la revista Academia. Revista sobre Enseñanza del Derecho}

Luego de aquella breve introducción sobre la creación de la revista Academia. Revista sobre Enseñanza del Derecho abordaremos sus alcances y objetivos, y nos detendremos en el análisis de algunos datos cuantitativos y cualitativos que arroja la producción de la revista. 
La revista hizo su aparición en el año 2003, y hasta la actualidad lleva publicados 19 números. Academia es distribuida en forma gratuita. El objetivo de Academia es constituirse en un espacio de intenso debate, rigurosa producción intelectual y amplia difusión, no sólo en el ámbito nacional sino latinoamericano hispano (y lusitano) parlante.

Ahora bien, veamos su contenido con suma atención. Del análisis de los índices de los 19 números publicados resulta que fueron publicadas 282 producciones. ${ }^{5}$ Las producciones aparecen en las siguientes secciones: "Prólogos", "Artículos", "Debates", "Estudios e Investigaciones", "Actualidad universitaria", "Clásicos", "Históricas", "Bibliográficas". La mayor cantidad de producciones originales y traducciones sobre enseñanza del Derecho se ubican en "Artículos", "Estudios e Investigaciones" y "Actualidad universitaria".

La sección "Artículos" responde a trabajos realizados por prestigiosos profesores de Derecho que, si bien se interesan por la enseñanza del Derecho, se acercan en general al tratamiento de la cuestión en forma esporádica. Dentro de este universo, 67 fueron escritos por varones, 24 por mujeres y 4 en grupo (mujer/varón). En este apartado se encuentra la mayor cantidad de traducciones de trabajos, escritas en inglés (en menor medida en alemán e italiano). En total se publicaron 95 trabajos.

La sección "Estudios e investigaciones" es, tal vez, más interesante para nuestro objetivo, ya que publica los trabajos de personas o equipos que desarrollan proyectos de investigación sobre enseñanza del Derecho o emparentados con la mencionada disciplina, y es mayormente resultado de la producción en universidades públicas argentinas. 23 corresponden a trabajos escritos por mujeres, 22 por varones, 11 en equipo conformados por varones y mujeres y dos como documento de una dependencia de la Facultad. En total se trata de 58 trabajos. La cantidad es menor que la de artículos y es claro que esto responde a que la cuestión "enseñanza del Derecho" aún es un campo de investigación poco explorado, con todo ha crecido y en forma considerable durante los últimos años ${ }^{6}$.

La sección "Actualidad universitaria" publica normas sobre planes de estudio, evaluación y acreditación de carrera, contenidos de las asignaturas, como así también documentos de trabajo e informes confeccionados por dependencias de las facultades. Así, 28 de los trabajos aparecen sin autor; 19 fueron escritos por varones, 8 por mujeres y dos en equipo (mujer/varón). Se trata de un total de 57 trabajos.

La sección "Clásicos" e "Históricos" recupera artículos y/o documentos ya publicados, en general por publicaciones de la Facultad, que siguen siendo de interés para discutir sobre la enseñanza del Derecho. Se publicaron 20 clásicos y 7 históricos, todos escritos por varones que se corresponde con la prácticamente nula presencia de mujeres en la docencia universitaria para fines de siglo XIX y hasta mediados del siglo $\mathrm{XX}^{7}$. Tres históricos se publican "sin autor" por corresponder a fallos.

\footnotetext{
${ }^{5}$ Los índices pueden ser consultados en:

http://www.derecho.uba.ar/publicaciones/rev_academia/index.php

${ }^{6}$ Cardinaux, N., "Presentación Academia 6", en Academia. Revista sobre Enseñanza del Derecho, Año 5

Número 9. 2007. P. 7-10.

${ }^{7}$ MacKinnon, C., "Integrando el feminismo en la educación jurídica", en Academia. Revista sobre

Enseñanza del Derecho, Año 3 Número 6. 2005. P.157-174.
} 
La sección "Bibliográficas" congrega reseñas o informes bibliográficos referidos a libros sobre enseñanza del Derecho o afines. 15 fueron realizadas por mujeres y 16 por varones.

La sección "Debates" se propuso generar intercambio de ideas en un mismo número y sobre un mismo tema. Tres fueron los debates, el primero sobre "ética académica", el segundo sobre "feminismo, géneros y currículum", y el tercero sobre las revistas jurídicas. En total se trata de seis artículos, tres escritos por mujeres y tres por varones.

Los prólogos corresponden a los números instituyentes de la revista: el primero escrito por el Decano de la Facultad de Derecho de la UBA en el 2003, el segundo escrito por la Vicedecana. Por lo demás, el tercero corresponde al número temático sobre género y currículum y el quinto realiza una suerte de recorrido (poético) sobre los números publicados hasta el 2007, diagnosticando que la revista generó para quedarse un espacio de discusión sobre enseñanza. Tres fueron escritos por mujeres y uno por un varón.

Respecto de los contenidos de los textos en general, se pueden observar seis grandes líneas de trabajo o interés. Un primer grupo aglutina los documentos sobre planes de estudio, reforma, actualización, planificación, evaluación, índices, acreditación de carrera. En general, son producciones de la Facultad que edita la revista; en algunos casos es el resultado de la discusión de la mesa de decanos de las facultades públicas argentinas. $^{8}$

Un segundo grupo de artículos se refiere a uno de los objetivos que generan fuertes desafíos para el desarrollo curricular: la integración de la investigación en el grado. Al respecto, se registran artículos que plantean la pregunta respecto de experiencias de asignaturas con el formato de taller sobre metodología de la investigación en un plan de estudios con estructura flexible en los últimos dos años de la carrera. ${ }^{9}$

\footnotetext{
${ }^{8}$ Resolución 1570/03 sobre actualización del plan de estudios (carrera Abogacía UBA); Resolución 1571/03 sobre el título de Profesor en Ciencias Jurídicas para el nivel medio (UBA); Estándares para la autoevaluación de la gestión institucional y las funciones de enseñanza-docencia, de investigación científica y de extensión universitaria de las facultades de Derecho de las universidades públicas; Resolución sobre contenidos mínimos de la carrera de Abogacía (UBA); Res. (CS) № 3797 reforma de los contenidos de la asignatura "Derecho" del Ciclo Básico Común (UBA); El nuevo plan de estudios por competencias de la Licenciatura en Derecho de la Universidad Autónoma de Baja California; Asociación Internacional de Escuelas de Derecho: elección de la doctora Mónica Pinto como presidenta; Simposio Institucional "Gestión del Cambio Curricular: el caso de la carrera de Abogacía". Presentación; Cambio y resistencia en la concepción y organización de los programas de Derecho (Germán Silva García); Vicisitudes de la reforma de la reforma curricular en la Facultad de Ciencias Jurídicas y Sociales de la Universidad de San Carlos de Guatemala (César Landelino Franco López); Presentación de la Carrera de Especialización y la Maestría en Derecho Internacional de los Derechos Humanos; Red Argentina de Posgrados en Educación Superior; Programa de Derechos Humanos 2007-2008; Notas sobre el Primer Congreso de Pedagogía Universitaria organizado por la Universidad de Buenos Aires (Scioscioli).

${ }^{9}$ Formación para la investigación en el campo del Derecho. Una aproximación a las Prácticas Educativas: la asignatura "Epistemología y Metodología de la Investigación" del Profesorado en Ciencias Jurídicas de la Facultad de Derecho de la Universidad de Buenos Aires (Orler); Historia del Derecho e investigación: una alianza estratégica (Kluger); La investigación jurídica aplicada enfocada a las realidades jurisdiccionales. Exigencia académica y social para los posgrados en Derecho (Zapata Bello); Filosofía de la evaluación de la universidad. Notas sobre metodología cualitativa en la investigación jurídicoeducativa (Galati); Un marco de comprensión del avance de la investigación jurídica en Colombia (Botero Bernal); Salir del neolítico. Investigación y enseñanza en las Facultades de Derecho (Tunc); Para una transformación de la Universidad. Nuevas relaciones entre investigación y docencia, de R. A. Barnett (ed.) (Ruiz); La articulación entre enseñanza e investigación del Derecho (Cardinaux); La enseñanza del
} 
Un tercer grupo de artículos reúne los trabajos que se ocupan de la formación jurídica y perfiles profesionales. Se refieren, por ejemplo, a la pregunta sobre qué perfil/es profesional/es genera la implementación de determinado plan de estudio. ${ }^{10}$ Otros se ocupan más específicamente de la enseñanza y los perfiles de jueces. Este contenido fue en aumento, a la par que creció el interés por los estudios de Posgrado sobre especialización en justicia o Maestría en magistratura. Por ello, se congregan en este grupo artículos que reflexionan sobre la cultura (y endogamia) judicial, sobre la función de juzgar, entre otros temas. ${ }^{11}$

Un cuarto grupo que se refiere también a un perfil profesional, la docencia, requiere, sin embargo, un apartado especial. Ellos son uno de los actores principales que ponen diariamente en juego el plan de estudios. Hay al respecto una fuerte preocupación sobre la evaluación docente y calidad docente, la formación docente y la ética académica, entre otros. ${ }^{12}$

Un quinto grupo aglutina los trabajos que se detienen en uno de los sujetos de la relación pedagógica: el estudiante. Esto genera especial interés porque muchas veces los

Derecho en la Universidad de Buenos Aires. Propuesta de investigación desde una perspectiva antropológica (Fischman y Seda).

${ }^{10}$ Cambios en la profesión jurídica en América Latina (Bergoglio); Una mirada jurídica crítica sobre el ejercicio de la Abogacía en el ámbito societario (Freeman); El problema de la discrecionalidad judicial (Kronman); Resolución del Parlamento Europeo sobre las profesiones jurídicas y el interés general en el funcionamiento de los sistemas jurídicos.

11 La Escuela Judicial: ¿una escuela para jueces? (Cardinaux/Clérico); Los oficios del jurista: la fragmentación de la profesión jurídica y la uniformidad de la carrera judicial (Binder); Segmentación en la profesión jurídica: cambios ocupacionales de los abogados argentinos, 1995-2003 (Bergoglio/Carballo); Las mujeres y la corte. La difícil implementación de la igualdad de sexos en el acceso a las magistraturas (Boigeol); ¿Dónde están las profesionales del Derecho en la Ciudad de Buenos Aires? (Kohen); Más mujeres en la justicia: los argumentos más frecuentes (Kohen); Protagonismo Judicial y representatividad política (Bergalli); La función de juzgar (Dalla Vía); Jueces o académicos: ¿A quiénes debemos mirar según nuestro Derecho Constitucional? (Kaufman) Júpiter, Hércules, Hermes: tres modelos de juez (Ost); El reformista y el conservador: un estudio sobre las actitudes judiciales (Reid); ¿Importa la ley? La respuesta de un juez al movimiento de la escuela crítica del Derecho (Rubin); El buen juez de primera instancia (Cueto Rúa); La enseñanza jurídica y el proceso de evaluación para la selección de funcionarios del Poder Judicial (Brigido/ Lista); Acervos de conocimientos y estructuras de interpretación. Un estudio microsocial del Poder Judicial del Estado de Puebla, México (Cuéllar Vázquez); Modelo de calidad para la planificación de la formación en una escuela judicial (Rodríguez Wagner); Entrenamiento de habilidades judiciales (Simon/Poritsky); Escuela judicial e Internet: la formación y capacitación judicial en la red (Scioscioli).

${ }^{12}$ Algunos resultados de la evaluación de la gestión docente de la Facultad de Derecho de la Universidad de Buenos Aires; La carrera docente como parte de la organización y de la formación de la docencia en la Universidad de Buenos Aires (Molinari/Ruiz); La enseñanza del Derecho según la evaluación de la docencia. Informe comparativo de los resultados obtenidos en los operativos de evaluación en los años 2003 y 2004 en la Facultad de Derecho (UBA) (Álvarez/del Río/Ruiz); Jornada de Enseñanza del Derecho ¿Por qué esforzarse por mejorar? Un comentario sobre la evaluación docente formal, sus efectos y alternativas (Acciarri); Evaluación de la calidad docente (Tolosa); Formar docentes reflexivos. Una experiencia en la Facultad de Derecho de la UBA (Anijovich y otras); La programación. Aportes para la enseñanza del Derecho (Ruiz/Tenutto); La evaluación de la enseñanza del Derecho. Análisis comparativo de los resultados obtenidos en los operativos de evaluación correspondientes a los años 2004 y 2005 (Ruiz); La formación universitaria en las diferentes áreas y profesiones: formación docente para la enseñanza universitaria. Una experiencia en la carrera docente de la Facultad de Derecho de la Universidad de Buenos Aires (Goggi/ Kolodny). 
estudios sobre formación jurídica se realizan fuertemente desde la perspectiva del proceso de enseñanza con el efecto de opacar el interés por el proceso de aprendizaje. ${ }^{13}$

Un sexto grupo se refiere a trabajos sobre modelos de enseñanza del Derecho: se trata de artículos que tienen una faz crítica que apunta a develar la forma en que se enseña el Derecho y una faz constructiva en la que se presentan propuestas algunas desestructurantes y otras que buscan reemplazar el modelo de enseñanza por otros ${ }^{14}$ con mayor presencia activa del estudiantado. Varios de los trabajos analizan modelos de enseñanzas en facultades locales y extranjeras; asimismo, aparecen algunos trabajos que lo analizan desde una perspectiva histórica. Otros trabajos abordan las bondades y desventajas de algunas estrategias de enseñanza y su relación con los modelos de formación jurídica. Aquí aparecen varios estudios sobre el método de casos en sus múltiples variantes ${ }^{15}$, las clínicas jurídicas y los servicios jurídicos gratuitos ${ }^{16}$ y en

${ }^{13}$ Características demográficas y sociales de los estudiantes de la Facultad de Derecho de la Universidad
de Buenos Aires; La presencia del mensaje educativo en la conciencia de los estudiantes: resultados de la
socialización en un modelo jurídico dominante (Lista/Begala); Orden social y socialización en la carrera
de Abogacía de la UNC: la perspectiva de los alumnos (Brígido/Lista); Conocer a los alumnos (Ubertone).

${ }^{14}$ Algunas sugerencias para escapar del silencio del aula (Böhmer); Volviendo a pensar sobre el enseñar y el aprender (Mariscal); Sobre las creencias de los juristas y la ciencia del Derecho (Carrió); El Derecho que debe enseñarse (Cardinaux/González); Distintos modelos de educación jurídica y las condiciones sociales en las que se apoyan (Gordon); La educación legal como preparación para la jerarquía (Kennedy); Maximizar el uso de modelos, minimizar el trabajo pesado, intensificar el aprendizaje: desarrollando y personalizando un sitio web extensible que enseña a los estudiantes de Derecho cómo entrevistar y aconsejar clientes (Le Brun); Comentarios que vale la pena hacer: la supervisión de trabajos académicos en las facultades de derecho (Fajans y Falk); Un Premio Nobel en la ciencia legal: teoría, trabajo empírico y el método científico en el estudio del Derecho (Ulen); Las condiciones de posibilidad de la ciencia jurídica en Antioquia (Botero Bernal); ¿De una facultad de Derecho hacia una Fachhochschule con nociones básicas de derecho? (Bull); La enseñanza del Derecho en Brasil (de Miranda Coutinho); La visión jeffersoniana de la educación jurídica (Douglas); Prometeo desencadenado: la enseñanza del Derecho en México (Flores); La formación de los juristas en el nacionalsocialismo (Frassek); Ciudadanía a través de la educación como objetivo europeo (Häberle); ¿Qué?, ¿Cómo? y ¿Para qué? Análisis y crítica al modelo tradicional de enseñanza del Derecho en México (Madrazo Lajous); Entre tradición e innovación: la formación de un jurista en Italia (Lupoi); Teoría y práctica en la enseñanza del Derecho (Pérez Lledó); Adiós a las revistas jurídicas (Rodell); La formación jurídica en procesos de integración regional: el caso de la Escuela de Derecho del MERCOSUR (Álvarez); A formaçao do jurista e o compromiso com a construçao da cidadania (Carlini); Politizar el aula (Kennedy); La enseñanza del derecho y la formación de los juristas en la primera mitad del siglo XX (Leiva); El pensamiento crítico: llaves, rutas y señuelos (Cardinaux y María Angélica Palombo); Escogiendo la ley (Goldfarb); Una comunidad de amigos y de extraños. Experiencia y construcción del discurso académico-jurídico (Otero); El Derecho en la cátedra y en el Tribunal (Nota sobre la enseñanza del Derecho) (Vázquez); La influencia de la educación jurídica en la formación valorativa de los abogados (Manzo); Aprender y enseñar en la Facultad de Derecho de la Universidad de Buenos Aires (Resnik); La interpretación "imposible" o el derecho como lenguaje escénico (de M. Albuquerque); La enseñanza del Derecho: ¿cofradía o archicofradía? (Bullard y Mac Lean); Autonomía (Guibourg); ¿Se puede estudiar la trompeta sin escucharla o verla? Sí claro: en la Facultad de Derecho (Marino); Educar para la democracia (Perícola).

${ }^{15}$ Notas sobre los libros de "casos" reconsiderados en el contexto del "método de casos" (Clérico); La Enseñanza del derecho constitucional en la república democrática (desde la perspectiva del método socrático) (Gelli); El análisis de casos como elemento imprescindible de la enseñanza jurídica (Goldschmit); Enseñar derecho pensando en el profesional del siglo XXI (Campari); Un buen juez es difícil de encontrar: un ensayo sobre el realismo legal y los libros de casos de las facultades de Derecho (Hayes); La enseñanza del caso "Marbury vs. Madison" (Garay); Por qué no enseñó "Marbury" (excepto a europeos del Este) y por qué ustedes tampoco deberían (Levinson) y nueve reseñas bibliográficas sobre libros de método de casos y análisis jurisprudencial.

${ }^{16}$ Algunas notas sobre el Patrocinio Jurídico Gratuito y el Curso de Práctica Profesional de la Facultad de Derecho de la Universidad de Buenos Aires (Scioscioli); Crónica de la Facultad: inauguración del 
mínima medida se trabaja sobre resolución alternativa de conflicto -ARD- y resolución de problemas. ${ }^{17}$

Asimismo, sólo en dos casos la relación Derecho y Cine es ocasión para reflexionar sobre una estrategia para la enseñanza del Derecho. ${ }^{18}$ En el apartado sobre modelos de enseñanza, cobra especial relevancia la pregunta sobre cómo integrar el feminismo y la cuestión de género en el currículum. ${ }^{19}$ También se observa una serie de artículos que sostienen que el modelo de enseñanza y de formación jurídica se mejora si el estudiante obtiene una mejor formación de base en Filosofía, Sociología y disciplinas afines. ${ }^{20}$ Por último, hay una producción considerable de trabajos que piensan la enseñanza del Derecho desde las estrategias o contenidos de sus respectivas asignaturas. ${ }^{21}$

Instituto de Enseñanza Práctica del Derecho (Lafaille); Actualidad sobre formación práctica en internet (Scioscioli); Algunos resultados del relevamiento estadístico del Servicio de Consultorio y Patrocinio Jurídico de la Universidad de Buenos Aires (Quiroga); Una espiral entre la teoría y la práctica: la ética del feminismo y la educación práctica (Goldfarb); La práctica profesional en la sede del CAREF-CELS del Patrocinio Jurídico Gratuito de la Facultad de Derecho de la Universidad de Buenos Aires (Scioscioli); La enseñanza clínica como recurso de aprendizaje jurídico (Witker); El caso "Clínica Junín": representación del conflicto y de la justicia en el discurso de los actores. Derechos sociales, democracia y ciudadanía (Gavernet); Resultados del relevamiento estadístico del Servicio de Consultorio y Patrocinio Jurídico Gratuito de la Universidad de Buenos Aires. Anuario 2006 (Quiroga/Scioscioli); La enseñanza de la práctica profesional. Una experiencia con futuro (Giavarino).

${ }^{17}$ Educación legal, estructuración de un curso introductorio de mecanismos alternativos de solución de conflictos en una facultad de Derecho colombiana (Varón Palomino); Desarrollando habilidades para resolver problemas legales (Nathanson); El papel de los abogados en la intención: la influencia de la práctica de la resolución alternativa de conflictos (ADR) sobre la educación y el pensamiento jurídicos (Fleerackers).

${ }^{18}$ El cine, ¿nos aporta algo diferente para la enseñanza del Derecho? (Thury Cornejo); La literatura y el cine como herramientas para la formación ética de los jueces (Ríos).

19 El género en la enseñanza del Derecho (Beloff/Clérico); El pensamiento feminista sobre el Derecho: un recorrido desde Carol Gilligan a Tove Stang Dahl (Facchi); Las enseñanzas y desafíos de hacerse caballeros (Guinier); Integrando el feminismo en la educación jurídica (Mackinnon); El feminismo en la enseñanza del Derecho en los Estados Unidos: estrategias para América Latina (Lemaitre Ripol); La enseñanza y el género en la Facultad de Derecho de La Plata (González/Salanueva); Feminismo, género y patriarcado (Facio/Fries); Constancias (Viturro); ¿Qué estoy haciendo cuando hago estudios de mujeres en los años noventa? (Stimpson); Proyecto "Transformación de la condición legal de la Mujer: integrando temas de género en la Doctrina y enseñanza del Derecho" (Saéz); El impulso de género en la enseñanza latinoamericana del Derecho (Red ALAS); Algunas sugerencias Bibliográficas (Bergallo/Di Corleto); Duración de los estudios universitarios en la carrera de Abogacía y diferencia de género (Gómez del Río/Ríos).

${ }^{20}$ La filosofía política en la formación del abogado (D'Auria); El Derecho y las Ciencias Sociales, especialmente la sociología (Llewellyn); Sobre la libertad de escribir (Moreno); El genial joven Moreno y la estructuración del nuevo Estado (Palacios); La enseñanza del discurso jurídico oral y escrito en la carrera de Abogacía (Álvarez); La Sociología del Derecho en la formación jurídica (Sieckmann); El uso y abuso de la filosofía en la enseñanza del Derecho (Nussbaum).

${ }^{21}$ Objetivos, contenidos y métodos en la enseñanza del Derecho Constitucional: algunas reflexiones (Treacy); La enseñanza de las Ciencias de las Finanzas en la Universidad de Buenos Aires desde su fundación hasta 1830 (Acevedo); Cánones en el Derecho Constitucional (Balkin/Levinson); Aportes para la postergada deconstrucción de la enseñanza del Derecho de familia (Herrera y Spaventa); Ética en internet. Nuevas herramientas para la enseñanza y aprendizaje de los contenidos éticos en la educación superior (Scioscioli); Derecho de la educación (Ciuro Caldani); Nociones sumarias sobre el estudio del Derecho Administrativo (Bielsa); El Derecho Constitucional y las especializaciones (Dalla Via); La enseñanza del concepto de Derecho desde la Filosofía del Derecho contemporánea (Laurrauri Torroella); El nuevo programa de Derecho Civil (Colmo); Las reformas en la Facultad de Derecho, método de estudio y enseñanza del Derecho Civil (Prayones); La enseñanza de "Teoría del Estado" en la Facultad de Derecho de la Universidad de Buenos Aires (Resnik y Perícola); En torno a la enseñanza de la criminología y las ciencias jurídicas en España: una coyuntura y un desafío en el fondo y en la forma (Abbott Matus); La percepción y configuración de los nuevos daños. Reflexiones sobre su inserción en la 
En suma, podemos conjeturar que la Revista estudiada es percibida por los docentes como un espacio de discusión sobre modelos de formación jurídica. Este tema tiene una fuerte presencia y aparece en forma recurrente. De forma complementaria, es importante también la cantidad de trabajos sobre contenidos y estrategias de enseñanza que se aplican o buscan aplicar en una asignatura determinada. En ambos casos son estos trabajos los que parecen estar dando pistas sobre la reflexión de los docentes sobre sus prácticas de enseñanza aunque no lo enuncien explícitamente. Ahora bien, estos artículos hablan de presencias de docentes de Derecho Constitucional, Teoría del Estado, Derechos Humanos, Derecho Internacional, Filosofía del Derecho, Sociología del Derecho o Metodología de la Investigación que reflexionan en esta línea; por lo contrario, son escasos los que lo hacen respecto de las asignaturas del Derecho Civil o Empresarial.

No se descarta que estos docentes reflexionen sobre sus propias prácticas pero en tanto no las expliciten (por ejemplo a través de publicaciones que la traten en forma más o menos directa) continuará vigente en el imaginario la hipótesis de enseñanza de un docente "dictando" contenidos (por lo general, del código o del "manual de cátedra") y de estudiantes que toman apuntes para luego estudiarlos (y repetirlos) en forma "memorística". No es ésta una conclusión que se desprenda directamente del caso aquí analizado pero la ausencia debe ser tan o más interpelada que la presencia y es ésta una conjetura acerca de sus razones.

Por otro lado, no debe descartarse la baja presencia de profesores de tiempo completo. En tanto los profesores trabajen principalmente en otra función jurídica (profesión legal, judicatura, ministerio público, asesorías, funcionarios, etc.) y tengan, además múltiples afiliaciones docentes (para comenzar, grado y posgrado), es difícil pensar que puedan tener el tiempo para realizar la reflexión que aquí se propone y publicar los resultados de esa reflexión.

Asimismo, no es un dato menor la circunstancia de que los análisis metodológicos en Derecho, en paralelo con los relacionados con la enseñanza práctica, no gozan de prestigio entre los académicos jurídicos ${ }^{22}$. Si se vincula este punto con el anterior, y en caso de que un profesor tenga tiempo libre para escribir, hay altas probabilidades de que lo haga sobre un tema que le reditúe mayores "beneficios" por lo menos simbólicos en el medio.

enseñanza jurídica (Garrido Cordobera); La sociedad abierta de los intérpretes constitucionales: una contribución para la interpretación pluralista y "procesal" de la Constitución (Häberle); Enseñanza de la técnica legislativa (Muro Ruiz); Enseñar Derecho Comparado en el siglo XXI: más allá de la dicotomía Derecho Civil / common law; Bases jurídicas de la educación para el desarrollo sostenible en el Mercosur (Novelli); Un nudo gordiano en la enseñanza del Derecho. A propósito de la integración de la filosofía jurídica en la dogmática y en la práctica jurídicas (García Jaramillo); Cuestiones y textos: sobre la enseñanza de la Teoría y la Filosofía del Derecho (de Prada García); Concepciones filosóficas y enseñanza del Derecho (Vázquez); Programa de Enseñanza de Derecho Internacional de los Refugiados; La enseñanza del Derecho Aeronáutico (Capaldo); El "diálogo" de las fuentes: fragmentación y coherencia en el Derecho Internacional contemporáneo (Do Amaral Júnior); La enseñanza del Derecho Procesal Constitucional. Experiencias y perspectivas (Loianno); Cultura y derecho constitucional. Entrevista a Peter Häberle (Ferreyra); La discriminación como objeto de estudios a nivel universitario. Una experiencia (Ubertone); Una nueva visita a la Filosofía del Derecho argentina (Atienza); El discurso jurídico recontextualizado en la Facultad de Derecho de la Universidad Nacional de Rosario. Análisis de dos materias de ciclo básico (Peseta).

${ }^{22}$ Goldfarb, P., "Una espiral entre la teoría y la práctica. La ética del feminismo y la educación práctica", en Academia. Revista sobre Enseñanza del Derecho, Año 3 Número 6. 2005. P.67-156. 
Una revista sobre enseñanza del Derecho requiere seguir planteándose el desafío, entre otros, de ampliar ese foro de debate a los docentes de las asignaturas tradicionalmente llamadas "dogmáticas" y predominantemente de Derecho Civil (por lo general, con fuerte presencia y de obligatoriedad en el currículum). Profundizado este proceso de reflexión se habrá ampliado el universo de discusión de las prácticas concretas de enseñanza, lo que ayudará a la implementación de los planes de estudio que buscan cambiar el núcleo duro de la matriz pedagógica obsoleta que aún siguen teniendo (en mayor o en menor medida) los modelos dominantes de enseñanza del derecho en varios países de América Latina y España.

\section{Referencias bibliográficas}

CARDINAUX, Nancy. "Presentación Academia 6", en Academia. Revista sobre Enseñanza del Derecho, Año 5 Número 9. 2007. P. 7-10.

GONZÁlEZ M.; Marano; BIANCO, C.; CORREA, M.; BERISSO, I. Estado del arte de la educación jurídica a diez años del Primer Congreso de Sociología Jurídica, ponencia presentada en el XI Congreso de Sociología Jurídica celebrado en la Facultad de Derecho de la Universidad de Buenos Aires, 2010, texto sin publicar.

GOLDFARB, P., "Una espiral entre la teoría y la práctica. La ética del feminismo y la educación práctica", en Academia. Revista sobre Enseñanza del Derecho, Año 3, Número 6. 2005. P. 67-156.

MACKINNON, C., "Integrando el feminismo en la educación jurídica", en Academia. Revista sobre Enseñanza del Derecho, Año 3, Número 6. 2005. P.157-174.

PARISE, A., "Las revistas jurídicas en el ámbito universitario: foros de expresión y laboratorios de escritura“, en Academia. Revista sobre enseñanza del Derecho, Buenos Aires, Número 15. 2010. P.123-132. 